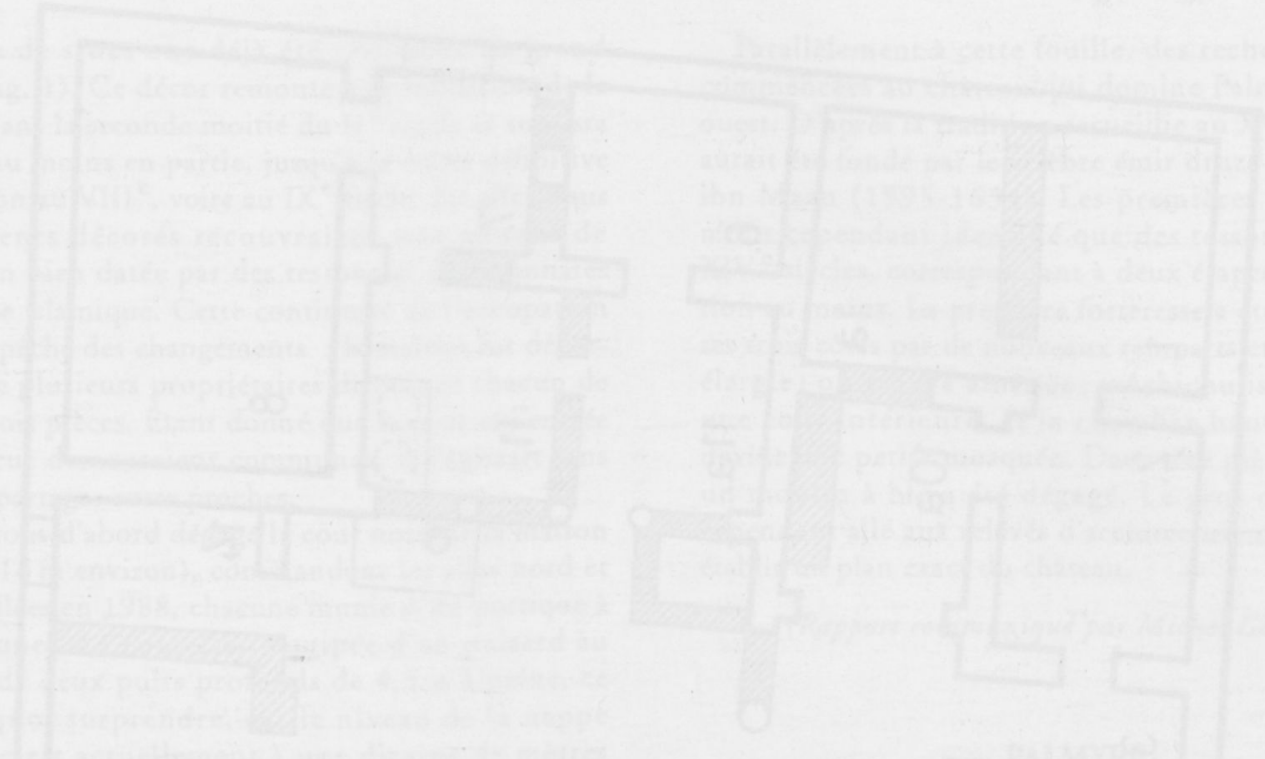

\title{
RAPPORTS DES MISSIONS ACTIVES À PALMYRE
}

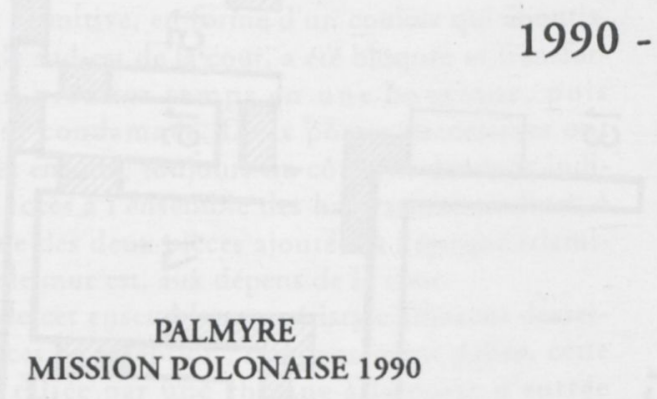

C'était la troisième saison consécutive consacrée à l'étude du quartier nord de la ville antique. Articulé par un réseau orthogonal de rues branchées sur la Grande Colonnade (section $A$, du Temple funéraire au Tétrapyle), ce quartier fut fondé, de toute apparence sur un terrain libre, à l'époque antonine, qui correspond à l'apogée de la prospérité caravanière de Palmyre devenue sous Hadrien une ville libre de l'Empire.

Les travaux de cette année ont touché deux îlots voisins, l'un résidentiel et l'autre occupé par une basilique civile convertie en une église. L'un et l'autre ont déjà été entamés lors des saisons précédentes (voir Syria 47/2 [1990], p. 449-451). Rappelons que la basilique présente du côté est une abside qui s'ouvrait sur la nef unique par un arc mouluré, datable de la seconde moitié du II ${ }^{\mathrm{e}}$ siècle. Nous avons procédé cette année au dégagement de l'extrados de cette abside, pour constater qu'elle avait été installée sur le sol d'une boutique adossée à la basilique dans son premier état; d'autres boutiques alignées sur celle-ci ont été en même temps fermées du côté de la rue et les murs de partition entre elles abolis pour former une salle accessible par le porche de l'église. Contrairement à ce que j'ai cru pouvoir affirmer l'année dernière, l'abside n'appartient donc pas, même dans ses fondations, au bâtiment primitif; le décor de l'ouverture percée dans le mur oriental de la basilique dut être emprunté à quelque monument démoli, selon la pratique que l'on a déjà pu constater au Camp de Dioclétien.

Le matériel recueilli en relation aux fondations de l'abside comprend notamment une monnaie de César Constance (324-337) et des tessons de l'époque. Il semble d'autre part probable que l'installation de l'église dans un bâtiment préexistant à vocation sans doute commerciale, trouve un reflet dans l'inscription gravée sur une colonne au-devant de la basilique : en 328, un fonctionnaire municipal du nom de Flavius Diogénès a restauré la toiture du portique de la Grande Colonnade correspondant à la largeur du monument. Si ce rapprochement est correct, l'église aurait été installée entre 324 (la conquête de l'Orient par Constantin) et 328 , lorsque la ville décida de relever les ruines de l'entrée du nouvel édifice de culte. Ce serait ainsi la première église de Palmyre, sans doute le siège épiscopal de Marinus, l'un des pères du Concile de Nicée.

Nous avons d'autre part continué la fouille d'une maison au nord-est de la basilique, où d'intéressants 


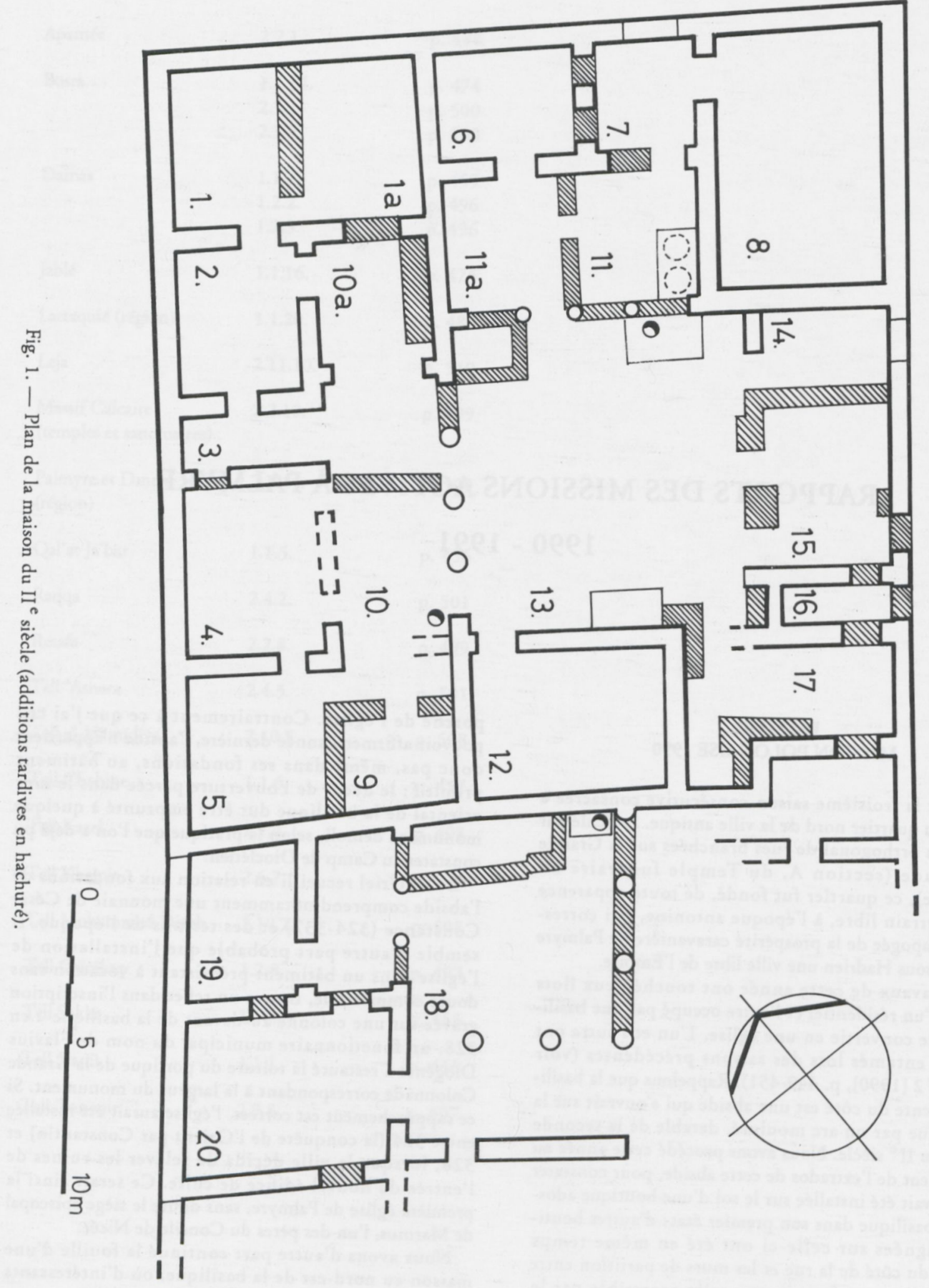


fragments de stucs ont déjà été retrouvés en grand nombre (fig. 1). Ce décor remonte à la fondation de la demeure dans la seconde moitié du II ${ }^{\mathrm{e}}$ siècle et subsista en place, au moins en partie, jusqu'à la ruine définitive de la maison au VIII ${ }^{\mathrm{e}}$, voire au IX $^{\mathrm{e}}$ siècle. En effet, tous les fragments décorés recouvraient une couche de destruction bien datée par des tessons et des monnaies de l'époque islamique. Cette continuité de l'occupation n'a pas empêché des changements : la maison fut départagée entre plusieurs propriétaires disposant chacun de deux ou trois pièces. Étant donné que la cour et l'entrée depuis la rue demeuraient communes, il s'agissait sans doute des partages entre proches.

Nous avons d'abord dégagé la cour nord de la maison ( $10 \mathrm{~m}$ sur $14 \mathrm{~m}$ environ), commandant les ailes nord et ouest fouillées en 1988 , chacune munie d'un portique à trois colonnes. La cour était équipée d'un puisard au milieu et de deux puits profonds de $4,5 \mathrm{~m}$ à peine, ce qui a de quoi surprendre, car le niveau de la nappe phréatique est actuellement à une dizaine de mètres plus bas. Un autre puits, trouvé juste à côté, a $8 \mathrm{~m}$ de profondeur.

L'entrée primitive, en forme d'un couloir qui aboutissait à l'angle sud-est de la cour, a été bloquée et transformée en un premier temps en une boutique, puis entièrement condamnée. Deux portes successives ont été ouvertes ensuite, toujours du côté est, donnant indirectement accès à l'ensemble des habitations tardives, à travers l'une des deux pièces ajoutées à l'époque islamique contre le mur est, aux dépens de la cour.

Au sud de cet ensemble, un péristyle adjacent desservait les pièces de réception; soigneusement dallée, cette cour était reliée par une chicane à la porte d'entrée mentionnée tout à l'heure, mais séparée par un mur continu de la partie privée de la maison, groupée autour de la cour nord. Le mur de partition étant, pour une raison qui nous échappe, tracé en biais, la cour dallée est irrégulière, et ses colonnes sont alignées sur deux côtés seulement, est et sud. Du côté ouest, on trouve deux pièces d'apparat, l'une s'ouvrant sous la colonnade, l'autre munie d'un porche à deux colonnes plus petites. Les deux salons étaient réunis par une porte dans le mur mitoyen et communiquaient avec une pièce encore non fouillée plus au sud. Des réfections, notamment le blocage des portes mitoyennes, des niches dans les parois et du porche, sont datables au début du VII siècle, d'après des monnaies retrouvées. Les deux pièces servaient encore, avec le reste de la maison, à l'époque islamique, avant que ne tombât le décor stuqué, ici particulièrement riche et comprenant une frise avec des motifs appliqués notamment des armes, des pieds de vigne, des oliviers, des têtes enfin, celles-ci abîmées par des iconoclastes. Il est devenu clair que la maison s'étendait encore au sud du secteur jusque là fouillé, avec au moins une troisième cour à colonnes.
Parallèlement à cette fouille, des recherches ont été commencées au château qui domine Palmyre au nordouest. D'après la tradition recueillie au XVIII ${ }^{\mathrm{e}}$ siècle, il aurait été fondé par le célèbre émir druze Fakhr ed-Din ibn Maan (1595-1634). Les premières prospections n'ont cependant identifié que des tessons du XIII ${ }^{\mathrm{e}}$ $\mathrm{XIV}^{\mathrm{e}}$ siècles, correspondant à deux étapes de construction au moins. La première forteresse a été entourée sur ses trois côtés par de nouveaux remparts et sensiblement élargie; on trouve ainsi des mâchicoulis donnant sur une cour intérieure, et la chambre haute d'une tour devint une petite mosquée. Dans une pièce du château un moulin à blé a été dégagé. Le gros de l'effort est cependant allé aux relevés d'architecture qui serviront à établir un plan exact du château.

\section{(Rapport communiqué par Michel Gawlikowski)}

\section{PALMYRE MISSION POLONAISE 1991}

Pendant cette quatrième saison consécutive occupée à fouiller le secteur du centre-ville au nord de la Grande Colonnade (voir Syria 67 [1990], p. 449-451), nous avons principalement continué les travaux dans la basilique et ses dépendances, ainsi que dans l'îlot d'habitation voisin. La fouille de ces deux ensembles n'est cependant pas terminée et nous ne pouvons fournir actuellement que des conclusions tout à fait préliminaires.

La grande maison à l'est de la basilique couvrait une surface bien supérieure à quelques $1000 \mathrm{~m}^{2}$ fouillés à ce jour. Nous avons dégagé, au cours de trois saisons, vingt quatre pièces disposées autour de deux cours à colonnes. Trois autres pièces, dont le contour des murs a été suivi, sont apparues comme intermédiaires entre la seconde cour déblayée et une troisième, plus au sud, dont le péristyle est partiellement debout. La maison occupait donc peut-être tout l'îlot (fig. 1).

Elle comportait, dans la partie fouillée, deux ensembles séparés et destinés à des usages différents. Autant les pièces groupées autour de la cour 13 forment de toute évidence les appartements de famille, autant la cour 22 desservait les pièces de réception et de séjour. Le mur qui sépare l'une de l'autre n'est percé d'aucune porte, de façon que la communication devait se faire par l'étage. Il est évidemment possible qu'il s'agisse en fait de deux maisons distinctes; cependant, la diversité apparente des fonctions nous fait pencher pour l'hypothèse d'une résidence unique, divisée en ce que l'Orient ottoman connaîtra sous les noms de haremlik et salemlik.

Les entrées respectives de ces deux ensembles sont contiguës et précédées d'une marche monolithique du côté de la rue. Chacune introduisait dans la cour qu'elle desservait par un passage en chicane, mais tandis que la 
cour d'apparat était approchée par un vestibule muni au fond d'une espèce de loge de concierge et par une pièce de transition (17 et 18 sur notre plan), l'entrée dans la cour de service se trouvait plus simplement sous l'escalier. En effet, l' "étroit couloir" 16 que nous avons trouvé muré de toutes parts, s'explique mieux comme l'espace sous un escalier montant depuis la cour à la terrasse; un autre escalier, semblable en tous points mais mieux conservé (loc. 25), a été retrouvé au coin de l'autre cour.

Selon les précisions stratigraphiques obtenues cette année par le démontage des contreforts tardifs dans la pièce 17, il a été possible de dater le blocage de deux entrées primitives, ainsi que l'abandon de l'escalier 16 et donc probablement de l'étage, au lendemain d'une destruction partielle par un tremblement de terre dans la seconde moitié du VI ${ }^{\mathrm{e}}$ siècle. C'était le début d'une transformation de la maison, partagée progressivement entre plusieurs habitations que nous connaissons dans leur-dernier état, celui du $\mathrm{IX}^{\mathrm{e}}$ siècle.

En effet, trois familles distinctes se sont installées autour de l'ancienne cour de service, chacune disposant de plusieurs pièces et séparée des voisins par des murets et des blocages. De même, la cour d'apparat a été partagée en deux pour accommoder encore deux autres habitations tardives. Cette mutation semble avoir été progressive et résulter tout naturellement d'une succession d'héritages, mais elle reflète néanmoins un profond changement des coutumes et du contexte social à la fin de l'Antiquité.

Le chantier principal cette année était cependant situé de l'autre côté de la rue qui délimite la maison à l'ouest et concernait une grande cour dépendant de la basilique. Celle-ci, accessible dans son dernier état par une porte du côté de la Grande Colonnade, s'ouvrait dès l'origine vers le nord-ouest par un triple portail donnant sur une cour qui se dessinait nettement en surface, même avant que ne commence la fouille. Nous avons réussi cette année à dégager une bonne moitié de cet espace mesurant $33 \mathrm{~m}$ sur $25 \mathrm{~m}$ et qui gardait la même largeur que la basilique elle-même avec ses dépendances (fig. 2).

La cour a été retrouvée encombrée des constructions tardives où de nombreux éléments de l'aménagement primitif avaient été réutilisés. Malgré ces déprédations, une bonne partie du dallage d'origine reste en place, ainsi que plusieurs bases de colonnes. Il sera donc possible de restituer l'aspect primitif de la cour, graphiquement et pour une part matériellement, une fois le dégagement complété.

Au fond de la cour, face à la basilique, est aligné un ensemble de pièces à destination incertaine, qui avaient subi plusieurs transformations et reconstructions au cours de leur longue existence entre la fin du II ${ }^{\mathrm{e}}$ et le $\mathrm{IX}^{\mathrm{e}}$ siècle. Il vaut mieux attendre que la fouille de ces installations soit terminée avant de proposer une explication de leurs phases successives.

Les trois autres côtés de la cour accueillaient un péristyle dont les côtés longs s'alignaient sur le portail de la basilique et dont le côté court, au-devant de celui-ci, était posé sur un perron surélevé au bout de trois marches qui montent depuis la partie basse de la cour. Toutes les colonnes de ce portique ont été jetées bas pour servir de fondations à des enclos et des habitations tardives.

La partie orientale de la cour est asymétrique par rapport à la nef de la basilique, parce qu'elle est alignée sur les boutiques et d'autres dépendances qui y étaient appuyées. Cette irrégularité a été annulée en y installant de ce côté une entrée distyle, une exèdre, également à deux colonnes entre pilastres, enfin une latrine publique placée entre les deux et accessible des deux côtés. L'entrée depuis la rue avait trois passages, dont celui du milieu plus large et plus haut, et constituait, à n'en pas douter, l'accès principal de l'ensemble basilical.

Tous les trois passages ont été trouvés bloqués, un four de potier installé derrière l'un de ces blocages, l'exèdre transformée en une étable, et toute la surface de la cour prise par des habitations rustiques. D'après le matériel céramique, cette occupation ne commença que dans la seconde moitié du VI ${ }^{\mathrm{e}}$ siècle, sans doute à la suite du même séisme qui endommagea la maison à côté, et survécut jusqu'au $\mathrm{IX}^{\mathrm{e}}$ siècle, tout comme cette même maison.

Dans la basilique, nous avons entrepris deux sondages dans l'intention de préciser la date de la conversion de ce monument en une église. Comme déjà proposé après la dernière saison, et confirmé cette année par de nouveaux tessons trouvés dans la couche relative à l'adjonction de l'abside, cette transformation remonte au IV $^{\mathrm{e}}$ siècle, peut-être à Constantin, comme le suggère une inscription de 328 (CANTINEAU, Inv. III, 27). En revanche, il est apparu que l'église a cessé de servir beaucoup plus tôt que nous n'avons d'abord cru, déjà à la suite du tremblement de terre du VI ${ }^{\mathrm{e}}$ siècle. À la différence de la cour voisine, la nef est demeuré à l'abandon depuis.

Cette conclusion s'est imposée lorsque nous avons trouvé dans l'abside, pratiquement à l'emplacement de l'autel, un puits muni d'un bassin remployé. Autant le puits est antique et servait déjà la boutique du II ${ }^{\mathrm{e}}$ siècle qui occupait cet endroit à un niveau plus bas, autant le vasque a été rajouté pour racheter la différence des niveaux, après le démontage partiel du dallage de l'abside. Il est évident que la découverte du puits et son réaménagement à un endroit pareil ne sont pas concevables dans une église en service.

D'autre part, nous avons étudié un monument identifié l'année dernière dans la Vallée des Tombeaux, face à la tour funéraire d'Atenatan du haut de laquelle on en 


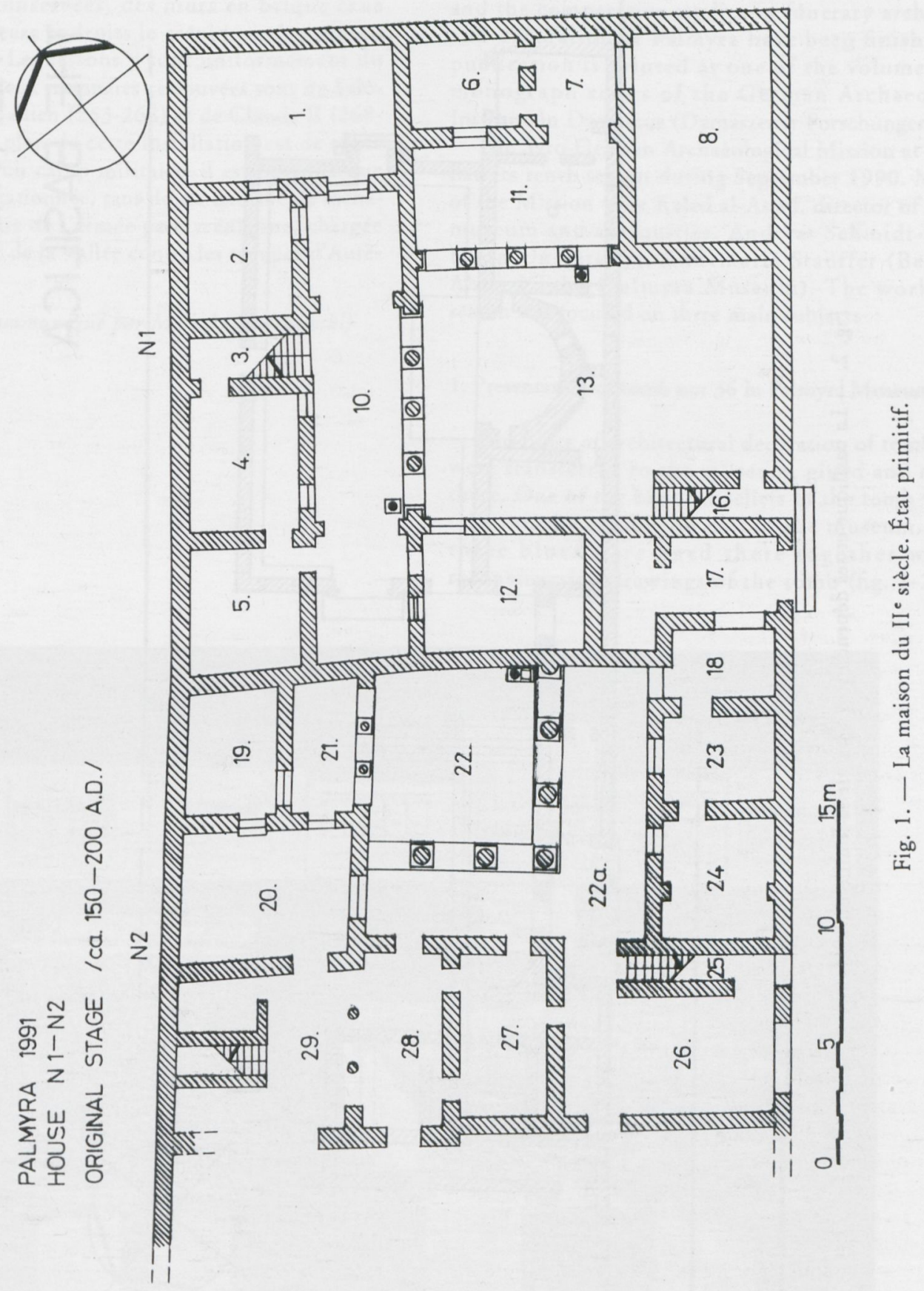




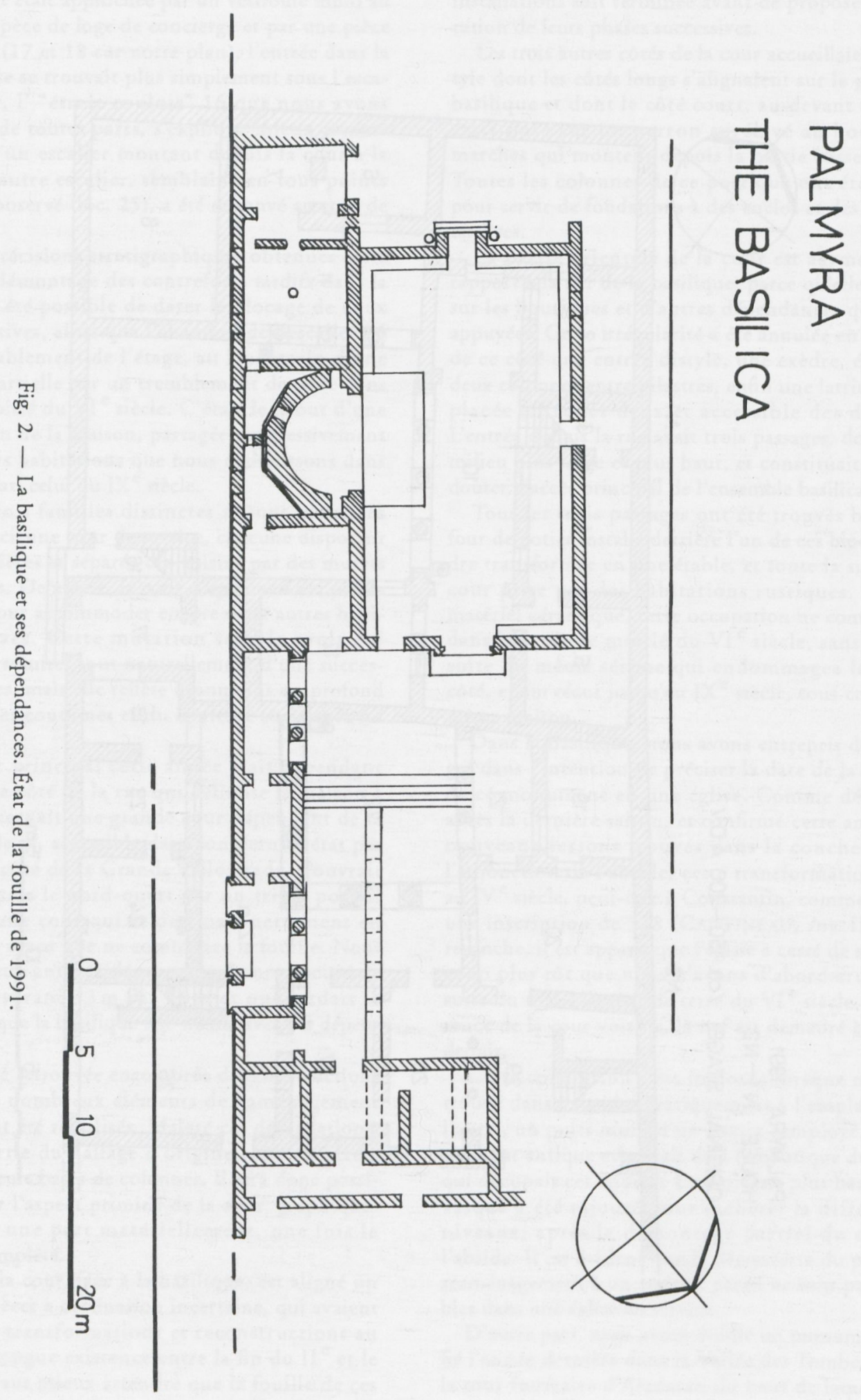


voit bien les contours. Il s'agit d'un enclos rectangulaire $63 \mathrm{~m}$ sur $48 \mathrm{~m}$, comprenant sur ses quatre côtés une rangée continue des pièces à peu près uniformes autour d'une grande cour au milieu. Sur les fondations en pierre partout conservées, des murs en brique crue gardent en plusieurs endroits le crépi jusqu'au niveau du sol moderne. Les tessons y sont uniformément du III ${ }^{\mathrm{e}}$ siècle et les deux monnaies retrouvées sont de Salonina, femme de Gallien (253-268) et de Claude II (268270). Comme le plan de cette installation est de toute évidence celui d'un camp militaire, il est possible que l'unité qui y fut stationnée, sans doute une aile de méharistes, faisait partie de l'armée palmyrénienne, chargée de garder le défilé de la Vallée contre les troupes d'Aurélien.

(Rapport communiqué par Michel Gawlikowski) 